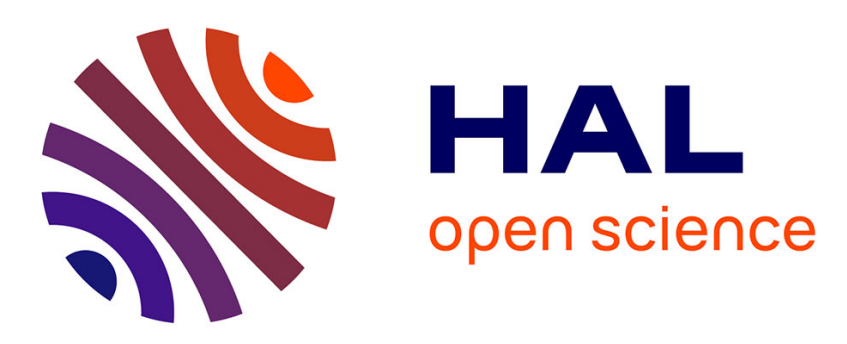

\title{
Adaptative delta-modulation coding for networked controlled systems
}

Fabio Gomez Estern, Carlos Canudas de Wit, Francisco Rubio, Jose Fornes

\section{To cite this version:}

Fabio Gomez Estern, Carlos Canudas de Wit, Francisco Rubio, Jose Fornes. Adaptative deltamodulation coding for networked controlled systems. ACC 2007 - American Control Conference, Jul 2007, New York City, United States. pp.7. hal-00130512

\section{HAL Id: hal-00130512 https://hal.science/hal-00130512}

Submitted on 12 Feb 2007

HAL is a multi-disciplinary open access archive for the deposit and dissemination of scientific research documents, whether they are published or not. The documents may come from teaching and research institutions in France or abroad, or from public or private research centers.
L'archive ouverte pluridisciplinaire HAL, est destinée au dépôt et à la diffusion de documents scientifiques de niveau recherche, publiés ou non, émanant des établissements d'enseignement et de recherche français ou étrangers, des laboratoires publics ou privés. 


\title{
Adaptive Delta-modulation Coding for Networked Controlled Systems
}

\author{
Fabio Gómez-Estern, Carlos Canudas-de-Wit, Francisco R. Rubio and José Fornés
}

\begin{abstract}
This paper investigates the closed-loop properties of the differential coding scheme known as Delta-Modulation $(\Delta-M)$ when used in feedback loops within the context of linear systems controlled through some communication network. We propose a new modified scheme of the original form of the $\Delta-M$ algorithm which improves the closed-loop properties. Semiglobal stability with convergence to a finite ball is proved in this framework, where the domain of attraction may be arbitrarily enlarged by tuning a quantization factor $\Delta$, in tradeoff with the precision at steady state. In a further step, parameter $\Delta$ is made adaptive, by defining an adaptation law exclusively in terms of information available at both the transmitter and receiver. With this approach, global asymptotic Stability of the Networked Controlled System is achieved for a class of unstable plants.
\end{abstract}

Index Terms-Differential coding, delta modulation, stabilization of linear systems in Networked controlled systems.

\section{INTRODUCTION}

This paper proposes an adaptive extension a of fixed-gain differential coding scheme previously introduced by the same authors (see [2]) in the context of linear systems interconnected through some transmission network. The problem is of interest in the area of Networked Controlled Systems (NCS), where we find several applications calling for datacompression algorithms aiming at reducing the amount of information that may be transmitted throughout the communication channel.

Delta modulation $(\Delta-M)$ is a well-known differential coding technique used for reducing the data rate required for voice communication, see [9]. The standard technique is based on synchronizing a state predictor on emitter and receiver and just sending a onebit error signal corresponding to the innovation of the sampled data with respect to the predictor. The prediction is then updated by adding a positive or negative quantity (determined by the bit that has been transmitted) of absolute value $\Delta$, a known parameter shared between emitter and receiver.

In [2] we have analyzed stability issues that appear when the (fixed-gain) $\Delta$-modulation scheme was embedded in a control system between the device measuring the state and the actuator. Delta modulation $(\Delta-M)$ algorithm can also be understood as the coarsest two-level (1-bit) quantizer. Thus, this technique is a simple alternative to works concerning the

C. Canudas-de-Wit is with The Laboratoire d'Automatique de Grenoble, UMR CNRS 5528, ENSIEG-INPG, B.P. 46, 38 402, ST. Martin d’Hères, FRANCE. Email: carlos.canudas-de-witeinpg.fr.

Fabio Gómez-Estern, Francisco R. Rubio and José Fornés, are with the Department of Automatic Control and Systems Engineering at the University of Sevilla. Email: rubio@esi.us.es, fornes@trajano.us.es, fabio@esi.us.es use of quantizers in the context of NCS, i.e. [5], [3], [8], [6], [10], [7], among others. Compared to these approaches, the modified form of the $(\Delta-M)$ algorithm proposed in [2], has the ability of providing a kind of separation principle, where the feedback gain if first designed assuming instantaneous unconstrained communication, and then the coding scheme is designed in order to preserve stability when embedded in the feedback loop. It is worth to remark that this modified form of the $\Delta-M$ coding structure explicitly contains information about the model plant and the controller feedback gain.

For a constant $\Delta$ it was shown in [2] that only a limited domain of attraction was obtained. In addition, the state was only guaranteed to converge asymptotically to a finite ball, begin its size related to the parameters of the open-loop plant, and on the the userdefined parameter $\Delta$.

By making $\Delta$ an adaptive quantity, more effective schemes of $\Delta$-modulation have been already proposed in the communication community [9]. The idea is to design an update law for $\Delta$, defined exclusively in terms of the information available both at the receiver and transmitter, aiming at improving the resolution of the differential coding by reducing the gain $\Delta$ for slowly varying signals, while enlarging $\Delta$ in case of rapid change of the input, and hence allowing for faster signal tracking and higher bandwidth of the transmitted signals. So far in the communication area, adaptation laws for $\Delta$ have been proposed under somewhat heuristic criteria, as little information is supposed to be available on the dynamics of the source signal. However, when dealing with feedback systems, the dynamical properties of the plant become very useful in designing the adaptive law. This problem, to which this paper is devoted, is framed as shown in Figure 1.

The problem of quantization with time-varying resolution in feedback loops has been addressed in [4] and [1]. The first of these works presents, in the case of fixed resolution, a scheme similar to [2], in the sense that the state estimation is computed trough a filter built upon the closed-loop system matrix. However, the extension to variable-scale quantization is only defined in the zooming-in direction, and hence the initial states are upper bounded by the initial choice of the zoom factor. As a consequence, only semi-global stabilization is achieved. Here we propose a $\Delta$-update law that works well for both in-and-out zooming directions, thus providing a means to capture unbounded initial states in the zooming-out stage and guaranteeing global asymptotic stability. Moreover, by defining an explicit $\Delta$-update law in both directions, if the state is driven temporarily out of the domain of attraction at any time due to unattended disturbances, the system will recover the stability, unlike the case of [4]. 


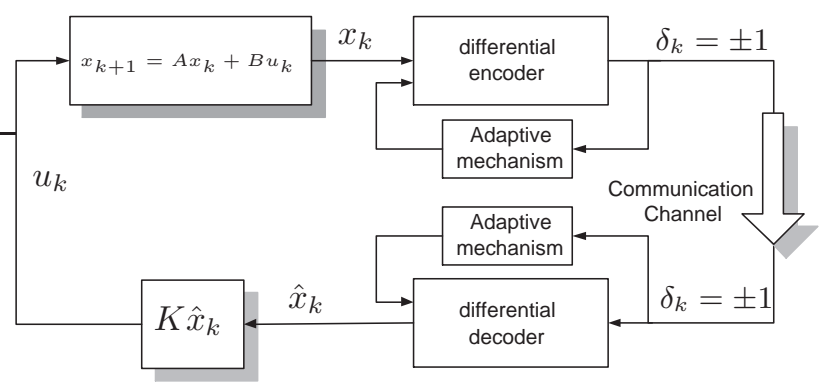

Fig. 1. Block diagram of the problem set up studied in this paper.

On the other hand, the work of [1] also guarantees global asymptotic stability with time-varying full-state quantization in two subsequent stages. Although our approach can be roughly understood as a particular case of the Theorems provided there, an important new feature introduced here is a state predictor that reduces the amount of data transmitted per sample by only sending the quantized prediction error. This reduces the data-rate to a minimum of 1 bit per sample, while the data-rate in Theorem 3 of [1] is $\log _{2}(2 M)$, a value that cannot be taken arbitrarily low. $M$ is actually defined as a function of the matrices $A, B, K$ of the control system, and it must be large enough to make the (thereby defined) scale factor small. In Theorem 4 of [1] a 1-bit per sample data-rate transmission scheme is also discussed, but here the separation principle is not present, as the feedback $u=H(q(x))$ is no longer a linear feedback of the state estimated on the receiver side. But probably the most significant difference between that approach and the one presented here is the zooming factor. In [1], it is calculated in terms of the convergence of the state to an attractive ellipsoid, whose geometry depends in a complex way on the system matrices. In our approach the zoom factor is updated at all times with a simple law that only depends on the last two state estimations, irrespective of the system matrices (and hence not subject to the side effects of bad identification).

First, we recall some of the results in [2] showing the main modification imparted to the standard $\Delta-M$ algorithms, and its resulting stability properties under fixed-gain $\Delta$. The main paper contribution is the introduction of an adaptation mechanisms consists of making time variant the quantization interval $\Delta$. This type of adaptation law, although well known in the communication field, is used and analyzed for the first time in the feedback configuration shown in Figure 1. It is also shown that this adaptive coding structure, modified as proposed in [2], is proven to yield closed-loop global asymptotic stability for a class of open-loop unstable linear systems.

\section{FIXED-GAIN $\Delta$ - $M$ CODING IN FEEDBACK}

In this section we recall some of the results in [2] which are pertinent for introducing the need for an adaptation law for $\Delta$. To make the presentation as simplest as possible, we just present the one-dimension noiseless case. In reference [2] readers can find the extension of this results to the ndimensional case, the consideration of noise, and the case of output feedback. These results concerns the case of fixed modulation gain, under the feedback configuration shown in Figure 1.

\section{A. Assumptions}

The hypothesis used in the results presented in this section, but also all along the paper, are the following:

- The transmitted information is binary $\delta_{k} \in\{-1,1\}$

- Only encoder-to-decoder information transmission is allowed (feedback between decoder to encoder is forbidden),

- Reliable noiseless channel transmission is considered (no data lost, or information distortion is considered),

- Transmission delays are neglected,

- Channel rate capacity is $R[b . p . s$.$] ,$

- Data is sent at a bounded rate (we select the sampling frequency in order to transmit only one $\delta_{k}$ at a time).

\section{B. One-dimension system example}

Consider the following one-dimensional discrete timesystem, together with the control law, and the differential coding modified law:

- Open-loop system, and encoder:

$$
\begin{aligned}
x_{k+1} & =a x_{k}+b u_{k} \\
\hat{x}_{k+1} & =[a-b K] \hat{x}_{k}+\Delta \cdot \delta_{k} \\
\delta_{k} & =\operatorname{sgn}\left(\tilde{x}_{k}\right)
\end{aligned}
$$

- Transmitted information: $\delta_{k} \in\{-1,1\}$,

- Control law and decoder:

$$
\begin{aligned}
\hat{x}_{k+1} & =[a-b K] \hat{x}_{k}+\Delta \cdot \delta_{k} \\
u_{k} & =-K \hat{x}_{k}
\end{aligned}
$$

with, $K \in \mathbb{R}, a \geqslant 1, a_{c}=(a-b K) ;\left|a_{c}\right|<1$, and $\tilde{x}_{k}=x_{k}-\hat{x}_{k}$.

The modified differential coder (2)-(4) differs from its standard form in that the term within the square brackets depends on the system model parameters $a$, and $b$, and on the control gain $K$. In the standard form this term is equal to one, i.e. the encoder is described by a delayed integral term. The advantages of this modification is that the coding error equations become decoupled from the system state equations, allowing for a simple analysis, and more important, for a design of the feedback gain $K$ independent to the coding structure (sort of separation principle). Therefore, the closedloop matrix $a_{c}$ can be designed by the sole and well known stabilization conditions for linear systems.

This algorithm gives the following error equations, with cascade structure:

$$
\begin{aligned}
& x_{k+1}=a_{c} x_{k}+b K \tilde{x}_{k} \\
& \tilde{x}_{k+1}=a \tilde{x}_{k}-\Delta \operatorname{sgn}\left(\tilde{x}_{k}\right)
\end{aligned}
$$

as $\left|a_{c}\right|<1$, stability of the whole system can thus be tacked by only studying the stability of the coding error equation (7), as is presented in Proposition 3 of [2]).

Below we provide a modified version of that Proposition where an additional restriction on parameter $a$ is considered. 
Theorem 1: $\Delta$ - $M$ ALgORITHM WITH FIXED-GAIN (MODIFICATION OF [2]). Consider system (1)-(5), with constant $\Delta$ and $a<2$. Then if the initial conditions of the coding error are such that

$$
\left|\tilde{x}_{0}\right|<r_{2}
$$

then the following hold:

- $\left|\tilde{x}_{k}\right|<r_{2}, \quad \forall k \geqslant 0$,

- $\exists k_{0}:\left|\tilde{x}_{k}\right| \leqslant r_{1} \forall k \geqslant k_{0}$, and

- $\lim _{k \rightarrow \infty} d\left(x_{k}, \mathcal{B}_{\gamma}\right)=0$.

where $r_{1}$ and $r_{2}$ are:

$$
r_{1}=\frac{a-1}{a^{2}-1} \Delta, \quad r_{2}=\frac{a+1}{a^{2}-1} \Delta
$$

and $d\left(x_{k}, \mathcal{B}_{\gamma}\right)$ is the minimum distance from $x_{k}$ to any point within the interval

$$
\mathcal{B}_{\gamma}:=\{x \in \mathbb{R}:|x|<\gamma\}, \quad \gamma=\frac{K b}{1-a_{c}} r_{1}
$$

Proof: The main argument can be read in [2], and the following analysis justifies the upper bound on $a$. Let $V_{k}=\tilde{x}_{k}^{2}$, and $\nabla V_{k}=V_{k+1}-V_{k}$. Then,

$$
\begin{aligned}
\nabla V_{k} & =\tilde{x}_{k+1}^{2}-\tilde{x}_{k}^{2} \\
& =\left(a^{2}-1\right) \tilde{x}_{k}^{2}-2 a \Delta\left|\tilde{x}_{k}\right|+\Delta^{2}
\end{aligned}
$$

This expression is negative on the region $r_{1}<\left|\tilde{x}_{k}\right|<r_{2}$. However, when $\left|\tilde{x}_{k}\right|<r_{1}, \nabla V_{k}$ takes positive values, up to a maximum of $\Delta^{2}$ at $\tilde{x}_{k 0}=0$. In that case, $\nabla V_{k}=\Delta^{2}$ and at the following sampling time $\left|\tilde{x}_{k 0+1}\right|=\Delta$. In order to ensure that the state remains within the region $\left|\tilde{x}_{k}\right|<r_{2}$ it is required that

$$
\Delta<r_{2}=\frac{a+1}{a^{2}-1} \Delta
$$

and this is satisfied if and only if $a<2$, so this is the condition required for asymptotic stability.

For some constant $\Delta$, this results means that if the initial condition $\left|\tilde{x}_{0}\right|$ are taken smaller than $r_{2}$, the error coding variable is locally attracted to a threshold delimited by the value of $r_{1}$, which depends linearly on the coding gain $\Delta$. This gains also delimits the stability border $r_{2}$, as shown the above expressions. Therefore, larger values for $\Delta$ will make the system more stable but less precise, and inversely reduction of the gain $\Delta$ will lead to small estimation error, but will at the same time reduce the domain where the system is keep stable.

This result displays an inherent trade-off between stability and precision when the gain $\Delta$ is fixed. This suggests the search for other coding strategies with variant gains. Note also that, as the sampling time ${ }^{1}$ is chosen small, $a$ approaches 1 and the precision is increased. Indeed, $\lim _{a \rightarrow 1} r_{1}(a)=0$, thus by making $T_{s}$ infinitely small, the limit case of the continuous-time infinite precision is approached.

\footnotetext{
${ }^{1}$ The pole of the discrete-time system is related to the pole, $\omega_{o l}$, of the open-loop continuous, one as $a=e^{\omega_{o l} T_{s}}$.
}

\section{AdAPTIVE $\Delta-M$ CODING SCHEME}

In this section we propose a $\Delta$-adaptation law resulting in a global asymptotic convergence of the estimation error and system states to zero. This is a significant achievement with respect to the fixed-gain scheme presented before which was limited to finite domains of attraction (though arbitrarily large) and convergence to finite balls.

\section{A. Adaptation law design}

Adaptive $\Delta$-modulation aims at improving the resolution of the differential coding scheme according to the size of the signals to be transmitted (in our case the states), hence a reasonable approach is to enlarge $\Delta_{k}$ for large values of the estimated states, and decrease it for smaller values. This is the main guideline for designing an adaptation law with the premise that the variations assigned to $\Delta_{k}$ should be defined exclusively in terms of the information available both at the receiver and transmitter; that is on $\delta_{k}$.

In order to design an $\Delta$-adaptive mechanism to achieve global asymptotic stability two opposite behaviors must be observed. These stem from the Theorem 1:

- For large values of the state $\tilde{x}$ when the condition for state decrease is not fulfill, i.e. $\left|\tilde{x}_{k}\right| \leqslant r_{2}, \Delta_{k}$ must grow monotonically at a higher rate than the plant escape velocity. This suggest an exponential growth law for $\Delta_{k}$ when the state is detected to be escaping from the origin.

- When the state is trapped into a domain of attraction $\left|\tilde{x}_{k}\right|<r_{2}, \Delta_{k}$ must decrease (for improving resolution) at a slower rate than the state convergence in order to preventing it from getting to small relative to the state and hence breaking again condition $\left|\tilde{x}_{k}\right|<r_{2}$ needed for convergence.

These behaviors indicate a trade-off design of the exponential reduction rate of $\Delta_{k}$ as compared to the potential growing (decreasing) rate of the open-loop plant. A key question underlying these issues is the way receiver supposed to distinguish between the cases where the error escaping or converging towards the origin. As $\tilde{x}$ is not available at the decoder side, the growth law will be designed on the basis of the transmitted data, i.e. $\delta_{k}=\operatorname{sgn}\left(\tilde{x}_{k}\right)$ and, possible, on its past values.

Based on the previous considerations, we propose an adaptive scheme with minimal storage and computation power requirements for updating $\Delta_{k}$ based on the following criteria:

1) If $\delta_{k}=\delta_{k-1}$ then the state is assumed to be escaping, thus $\Delta_{k}$ must be increased.

2) If $\delta_{k} \neq \delta_{k-1}$ then the state is assumed to converge (oscillating close to zero) and $\Delta_{k}$ must be decreased.

The following update law is proposed:

$$
\begin{aligned}
\Delta_{k+1} & =\phi_{k+1} \Delta_{k} \\
\phi_{k+1} & =\lambda^{-}+\frac{1}{2}\left(\lambda^{+}-\lambda^{-}\right)\left|\delta_{k+1}+\delta_{k}\right|
\end{aligned}
$$

where $0<\lambda^{-}<1$ is exponential decay rate of $\Delta_{k}$, and $\lambda^{+}>1$ is exponential growth rate. This adaptation law can 
be seen as a generalization of the (1970) adaptation Jayant's rule which can be consulted in [9]. The Jayant's rule is described by $\Delta_{k+1}=\lambda_{0}^{\delta_{k+1} \delta_{k}} \Delta_{k}$, with $\lambda_{0} \geqslant 1$. Hence this is a special case of our proposed rule with $\lambda^{+}=\lambda_{0}$, and $\lambda^{-}=1 / \lambda_{0}$. As it will be seen later, allowing $\lambda^{+}$and $\lambda^{-}$to be freely choosing, the stability conditions turnout to be less conservative.

\section{B. Error equations}

The complete feedback systems with the adaptive delta $\Delta$-modulation coding scheme is then:

- Open-loop system (10), and encoder (11)-(13):

$$
\begin{aligned}
x_{k+1} & =a x_{k}+b u_{k} \\
\hat{x}_{k+1} & =[a-b K] \hat{x}_{k}+\Delta_{k} \cdot \delta_{k} \\
\Delta_{k+1} & =\phi_{k+1} \Delta_{k} \\
\phi_{k+1} & =\lambda^{-}+\frac{1}{2}\left(\lambda^{+}-\lambda^{-}\right)\left|\delta_{k+1}+\delta_{k}\right|
\end{aligned}
$$

- Decoder (14)-(16) and control law (17):

$$
\begin{aligned}
\hat{x}_{k+1} & =[a-b K] \hat{x}_{k}+\Delta_{k} \cdot \delta_{k} \\
\Delta_{k+1} & =\phi_{k+1} \Delta_{k} \\
\phi_{k+1} & =\lambda^{-}+\frac{1}{2}\left(\lambda^{+}-\lambda^{-}\right)\left|\delta_{k+1}+\delta_{k}\right| \\
u_{k} & =-K \hat{x}_{k}
\end{aligned}
$$

With the above definitions, the closed-loop error dynamics become

$$
\begin{aligned}
\tilde{x}_{k+1} & =a \tilde{x}_{k}-\Delta_{k} \cdot \delta_{k} \\
\Delta_{k+1} & =\phi_{k+1} \Delta_{k}, \quad \Delta_{0}>0 \\
\phi_{k+1} & =\lambda^{-}+\frac{1}{2}\left(\lambda^{+}-\lambda^{-}\right)\left|\delta_{k+1}+\delta_{k}\right|
\end{aligned}
$$

The causality of the system is guaranteed by the fact that all times the computation of $\tilde{x}_{k+1}$ is only based on the $\tilde{x}_{k}$ and older values. The following Proposition states the stability of the closed-loop system

\section{Main result (stability analysis)}

Theorem 2: The error trajectories $\tilde{x}_{k}$ of system (18), resulting from the adaptive $\Delta$-modulation coding scheme (10)(17), globally asymptotically converge to zero as $k \rightarrow \infty$ if there exist parameters $\lambda^{+}>1, \lambda^{-} \in(0,1)$ satisfying the following inequalities:

$$
\begin{aligned}
& \lambda^{+}>a \\
& \lambda^{-}<\left(\lambda^{+}\right)^{-\frac{\beta}{2}},
\end{aligned}
$$

where

$$
\begin{aligned}
\beta\left(a, \lambda^{-}, \lambda^{+}\right) & \triangleq 1+\log _{\rho}\left(1+\frac{a\left(a-\lambda^{-}\right)(\rho-1)}{\left(\lambda^{-}\right)^{2}}\right) \\
\rho & \triangleq \frac{\lambda^{+}}{a},
\end{aligned}
$$

Moreover, $\Delta_{k}$ also converges to zero regardless it initial value $\Delta_{0}$.
Proof: The claim will be proved in two steps. First, a new variable is defined in order to capture the proportion between $\tilde{x}_{k}$ and $\Delta_{k}$, namely

$$
y_{k} \triangleq \frac{\tilde{x}_{k}}{\Delta_{k}}
$$

and boundedness of that variable will be proved. Secondly, it will be shown that $\Delta_{k}$ asymptotically converges to zero. Consequently, convergence of $\tilde{x}_{k}$ to the origin is directly implied.

Along the trajectories (18), variable $y_{k}$ evolves along the dynamics

$$
y_{k+1}=\frac{1}{\phi_{k}}\left(a y_{k}-\operatorname{sgn}\left(y_{k}\right)\right) \text {. }
$$

Fact 1. Trajectories of $y_{k}$ cross the zero axis in finite time.

This means that starting from any initial condition, $\tilde{x}_{0}$ and $\Delta_{0}$, and thus $y_{0}$, there must be a future time $k_{0}<\infty$ such that $y_{k_{0}-1} \cdot y_{k_{0}}<0$. This is easily shown by imagining a trajectory with no zero crossings on $y_{k}$ (hence on $\tilde{x}_{k}$ as $\Delta_{k}$ is always positive). Assuming initially positive $\tilde{x}$, i.e. starting from $y_{0}>0$, we have,

$$
y_{k+1}=\frac{1}{\lambda^{+}}\left(a y_{k}-1\right),
$$

hence

$y_{k+1}-y_{k}=\frac{1}{\lambda^{+}}\left(a y_{k}-1\right)-y_{k}=\left(\frac{a}{\lambda^{+}}-1\right) y_{k}-\frac{1}{\lambda^{+}}<-\frac{1}{\lambda^{+}}$

for the given choice of $\lambda$. Then, starting from $y_{0}>0$, we have

$$
y_{k}<y_{0}-\frac{k}{\lambda^{+}}
$$

and hence there is some constant $k_{0} \leqslant y_{0} \lambda^{+}$such that $y_{k_{0}-1}>0$ and $y_{k_{0}}<0$. Due to the symmetry of the system equations, a similar argument applies if the trajectory starts from $y_{0}<0$.

\section{Fact 2. Trajectories of $y_{k}$ are bounded after finite time.}

Immediately after a zero crossing of the system (assuming + to - without loss of generality), $y_{k_{0}}$ is bounded as follows

$$
y_{k_{0}}=\frac{1}{\lambda^{+}}\left(a y_{k_{0}-1}-1\right)>-\frac{1}{\lambda^{+}}
$$

hence $\left|y_{k_{0}}\right|<\frac{1}{\lambda^{+}}$, as $y_{k_{0}-1}>0$ and $y_{k_{0}}<0$. Now if we search for bounds on subsequent samples we must update the growth factor of $\Delta$ to $\lambda^{-}$and compute

$$
y_{k_{0}+1}=\frac{1}{\lambda^{-}}\left(a y_{k_{0}}+1\right)
$$

and it is easy to see that the the right hand side of the last expression is positive, as from the bound $0>y_{k_{0}}>-1 / \lambda^{+}$ we have

$$
y_{k_{0}+1}>\frac{1}{\lambda^{-}}\left(-\frac{a}{\lambda^{+}}+1\right)>0 .
$$

Moreover, $\left|y_{k_{0}+1}\right|<1 / \lambda^{-}$. These observations are summarized in Fig. 2, where the necessity of double zero crossing after a set of positive values is illustrated, as well as the upper bounds inferred by the switching dynamics. 


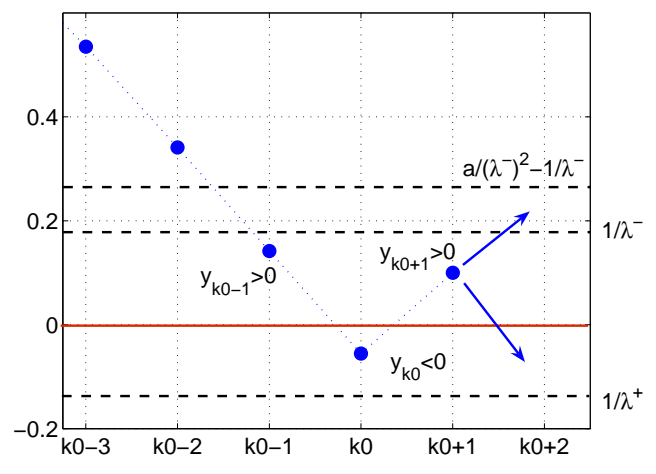

Fig. 2. Behavior of $y_{k}$ at zero crossings.

In order to conclude the analysis on the bounds, one step further must be taken on the switching dynamics. Using $0<$ $y_{k_{0}+1}<1 / \lambda^{-}$, we have

$$
y_{k_{0}+2}=\frac{1}{\lambda^{-}}\left(a y_{k_{0}+1}-1\right),
$$

and hence

$$
-\frac{1}{\lambda^{-}}<y_{k_{0}+2}<\frac{1}{\lambda^{-}}\left(\frac{a}{\lambda^{-}}-1\right),
$$

As illustrated in Fig. 2, two situations (a) and (b) are then identified,

(a) $-\frac{1}{\lambda^{-}}<y_{k_{0}+2}<0$. This situation (including the norm bound) is exactly the one found at instant $k_{0}+1$, then it has been already considered

(b) $y_{k_{0}+2}>0$. In this case we have two subsequent positive samples, i.e. the situation of $y_{k_{0}-1}$ is recovered. In that case, the dynamic equation turns into (23) and along it the map $y_{k} \rightarrow y_{k+1}$ is contracting, hence the norm will be decrease until a future sign change.

The previous analysis yields that after the first sign change, the state $y_{k}$ remains bounded as

$$
\left|y_{k}\right|<\frac{1}{\lambda^{-}}\left(\frac{a}{\lambda^{-}}-1\right)
$$

With the above facts, the proof of the proposition reduces to show asymptotic convergence to zero of $\Delta_{k}$, and hence concluding convergence o the state $\tilde{x}$. With this objective we will use the following definition,

Definition 3.1: Given a sequence of positive (negative) samples of $y_{k}$, the fly-time is the number of sampling instants elapsed between the two zero-crossings that enclose the signal. For its computation, the first and the last positive (negative) samples are considered.

This magnitude is viewed in Fig. 3. We will compute a non-conservative upper bound on it. Indeed, considering $y_{0}$ as the first positive sample (i.e. resetting the time count), we have along the dynamics (23),

$$
y_{k}=\rho^{k}\left(y_{0}-\frac{1}{a}\left(\frac{\rho^{k}-1}{\rho-1}\right)\right)
$$

then, the zero crossing occurs at the next sampling instant

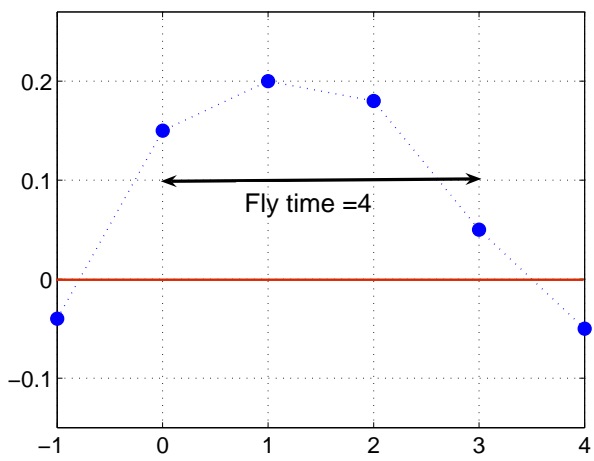

Fig. 3. Definition of fly-time.

after the time the right hand side of this equation vanishes. Therefore, the fly-time $k^{*}$ is bounded as

$$
\begin{aligned}
k^{*} & \leqslant 1+\log _{\rho}\left(1+y_{0} a(\rho-1)\right) \\
& \leqslant 1+\log _{\rho}\left(1+\frac{a\left(a-\lambda^{-}\right)(\rho-1)}{\left(\lambda^{-}\right)^{2}}\right)=\beta\left(a, \lambda^{-}, \lambda^{+}\right)
\end{aligned}
$$

for which we have used the finite-time bound on $y_{k}$ (and hence $\left.y_{0}\right)$ computed in (25).

On the other hand, the fly-time can be regarded as the exact number of times the $\Delta$ factor is increased via the exponential law $\Delta_{k+1}=\lambda^{+} \Delta_{k}$, then the net value of $\Delta$ after the flying period, starting from $\Delta_{0}$ is

$$
\Delta_{k}=\left(\lambda^{+}\right)^{k} \Delta_{0},
$$

Hence a condition for asymptotic convergence of $\Delta_{k}$ to zero is that, on zero crossings, the net decrease in $\Delta$ compensates the net amount increase over the flying period. This is guaranteed by choosing $\lambda^{-}$such that, considering $k_{0}$ as the first negative sample (again as in Fig. 2)

$$
\Delta_{k 0+2}=\left(\lambda^{-}\right)^{2} \Delta_{k 0}<\left(\lambda^{-}\right)^{2}\left(\lambda^{+}\right)^{\beta} \Delta_{0}
$$

where the power in $\left(\lambda^{-}\right)^{2}$ has been introduced using the fact that after a flying period, two consecutive zero crossings must occur (see argument of Fact 2). This gives a less restrictive condition on $\lambda^{-}$. Hence, the condition of net decrease of $\Delta$ after a flying period is

$$
\Delta_{k 0+2}<\Delta_{0} \Leftarrow\left(\lambda^{-}\right)^{2}\left(\lambda^{+}\right)^{\beta}<1,
$$

or, equivalently,

$$
\lambda^{-}<\left(\lambda^{+}\right)^{-\frac{\beta}{2}}
$$

which is the condition (20) stated at the Proposition.

¿From the net convergence of $\Delta_{k}$ to zero and the boundedness of $y_{k}$, we conclude that

$$
\lim _{k \rightarrow \infty} \tilde{x}_{k}=0
$$

This completes the proof. 


\section{PARAMETER VALIDITY REgION}

The choice of parameters $\lambda^{+}$and $\lambda^{-}$fulfilling the stability conditions (19)-(20) is nontrivial. Indeed, (20) is an implicit equation where $\lambda^{-}$appears both at the left and the right hand sides, and the solvability depends on the particular value of $a$.

Defining $\psi\left(\lambda^{+}, \lambda^{-}, a\right) \triangleq \lambda^{-}-\left(\lambda^{+}\right)^{-\frac{\beta}{2}}$ from (20), it is clear that there is a set of valid parameters if and only if $\psi(\cdot)$ becomes negative somewhere in the $\mathbb{R}^{2}$ region

$$
\left[\lambda^{-} \in(0,1)\right] \times\left[\lambda^{+} \in(a, \infty)\right],
$$

however, some calculations that will be presented elsewhere show that any solution of (20) must lie within the set $\Omega_{a}$ : $\left[\lambda^{-} \in(0,1)\right] \times\left[\lambda^{+} \in\left(a, \lambda_{\max }^{+}(a)\right)\right]$, where

$$
\lambda_{\text {max }}^{+}(a) \triangleq a \frac{1+\sqrt{1+\frac{4}{a(a-1)}}}{2},
$$

For a given value of the open-loop pole $a$, if there is no pair $\left(\lambda^{+}, \lambda^{-}\right) \in \Omega_{a}$ making $\psi(\cdot)$ negative, then there is no valid solution for (19)-(20). Therefore, $\Omega_{a}$ is the region that should be numerically scanned for solutions.

Unfortunately, for some values $a$ there is no such solution, while, as can be easily calculated, the condition $a<1.313$ guarantees the existence of solutions.

Fig. 4 (left) clearly shows that for $a=1.2$ there is a set of values of $\lambda^{+}$and $\lambda^{-}$for which the plotted expression is negative, i.e. the conditions are fulfilled. On the other hand, for values of $a \geqslant 1.313$ ( $a=1.4$ in Fig. 4) (right), nothing can be said about the existence of admissible parameters, but it can be observed that the whole surface $\psi$ is above zero and no solution has been found numerically. Moreover, as the right hand side of (20) decreases with $a$, there will be no more solutions for greater $a$.

\section{RELATION WITH THE N \& S CONDITION FOR STABILIZATION UNDER CHANNEL LIMITATIONS.}

A further issue that must be addressed with respect to the adaptive $\Delta-M$ scheme is the question wether the limitation on the open-loop poles (parameter $a$ ) to be below 1.6180 is a structural property or a limitation due to the sufficient nature of the result.

In any case, our limitation should be consistent with the necessary and sufficient condition for stabilization presented in [11]. This condition indicates that the minimal data rate required for stabilizing a discrete-time system via a communication channel of maximum rate capacity $R$ [b.p.u. $]^{2}$ is related to the unstable open-loop poles $\left(\lambda_{i}^{u n}\right)$ as: $R>$ $\sum \log _{2}\left(\lambda_{i}^{u n}\right)$, which in our case simplifies to:

$$
R>\log _{2} a
$$

The implicit assumption made within the framework of our discrete-time formulation is that the channel can reliably transmit one bit-per-unit of time, that is that $R=1$. This means that with regard to the condition (27), the maximum

\footnotetext{
${ }^{2} R$ is given in dimensionless units, i.e. bits per unit of time [b.p.u.]
}
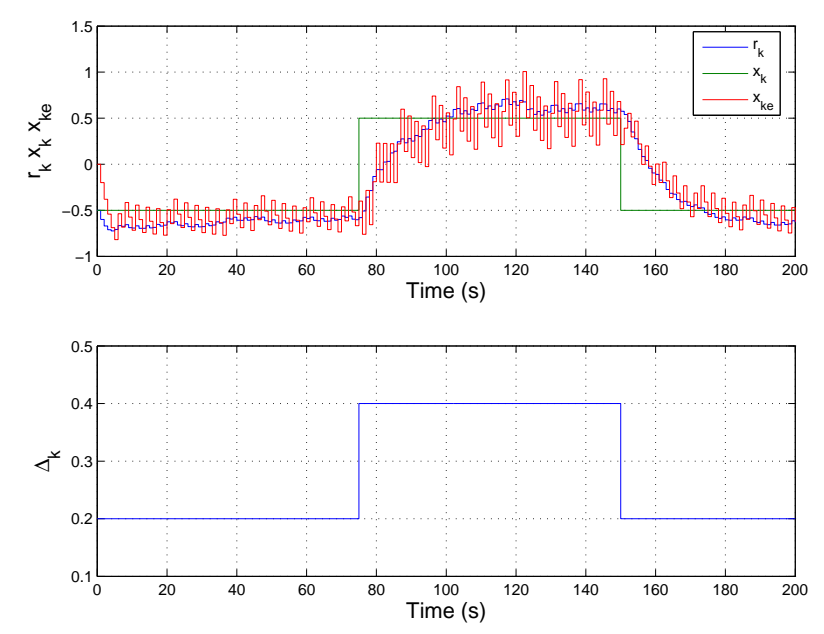

Fig. 5. Simulation results for non-adaptive $\Delta$ - $M$ scheme, with $\Delta$ fixed along specific intervals.

admissible value for $a$ is $a<2$, which is consistent with our sufficient condition $a<1.313$, probably due to the technicalities used for the stability analysis, or either due to the particular structure of the proposed adaptation law. This also indicates that there is some conservatism in the computation of the admissible set of the parameter $\lambda^{+}$and $\lambda^{-}$. Some alternative adaptation strategies could be devised in order to improve this bounds. For example, a mores sophisticated adaptive algorithm based on more past samples of $\delta_{k}$ could be possible designed.

\section{Simulations}

System (10)-(17) has been simulated for the set of values $a=1.1, b=1, K=0.2, x_{k}(0)=-0.5, \hat{x}_{k}(0)=0$, with $\Delta_{0}=5, \lambda^{-}=0.4, \lambda^{+}=1.21$, according to conditions (19) and (20).

For the sake of comparison with the non-adaptive scheme presented in [2], a first simulation has been made with a constante value of $\Delta$. Fig. 5 shows the behavior of the closed-loop system when the $\Delta$ is given fixed values, (it is only changed at the times the set-point changes). As can be seen, the granularity of the closed-loop error signal is directly related to the choice of the constant $\Delta$. During the simulation, after the first step change of the reference, $\Delta$ has been made larger and the granularity significantly increases. The main issue of [2] was that $\Delta$ cannot be fixed at a very low value (thus reducing the granularity) without compromising the domain of attraction.

Fortunately, this has been successfully tackled with the new adaptive approach, as is illustrated in Fig. 6. In the upper plots of this figure, the state $x_{k}, \hat{x}$ and the set-point $r_{k}$ are depicted, while the adaptive quantization parameter $\Delta_{k}$ is plotted on the lower graph. As expected, convergence to zero is obtained in all cases. The granularity in the steady state is due to the fact that $\Delta_{k}$ has not been allowed to take values below $\Delta_{\min }=0.05$. Without this saturation, $\Delta_{k}$ would keep tending to zero in steady state, and whenever a disturbance drives the state away from the origin, a large number of 

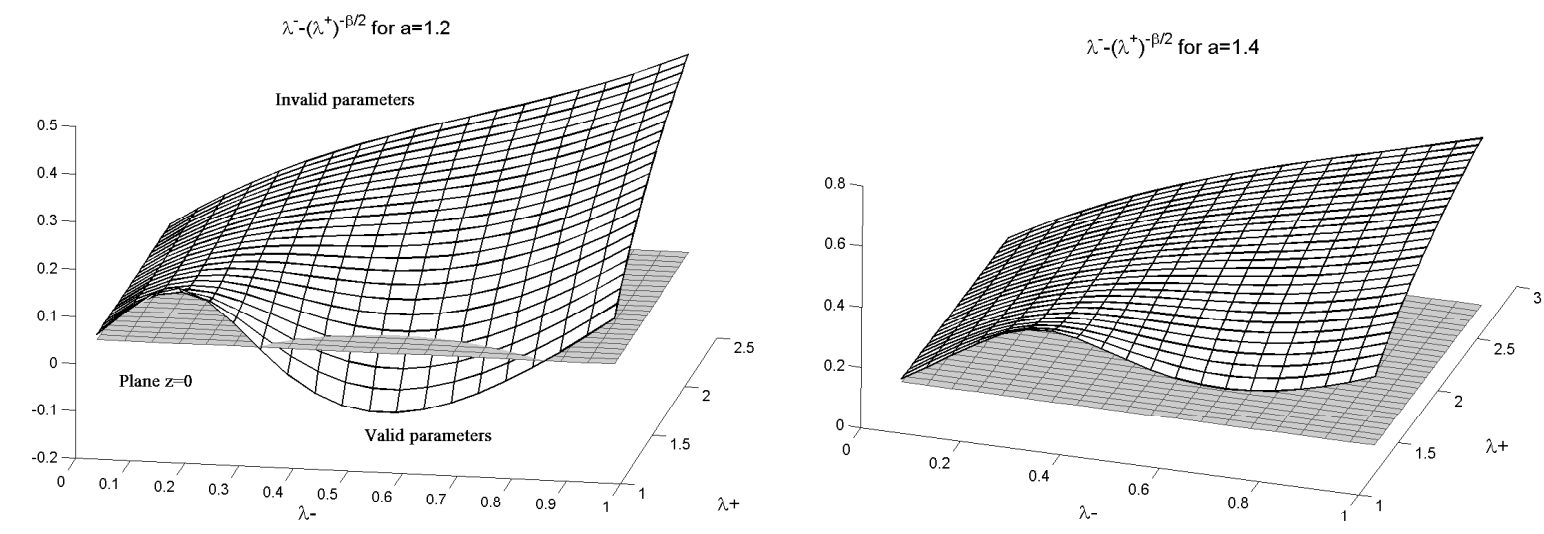

Fig. 4. (Left) Expression $\lambda^{-}-\left(\lambda^{+}\right)^{-\frac{\beta}{2}}$ for $a=1.2$; (Right) The same expression for $a=1.4$.
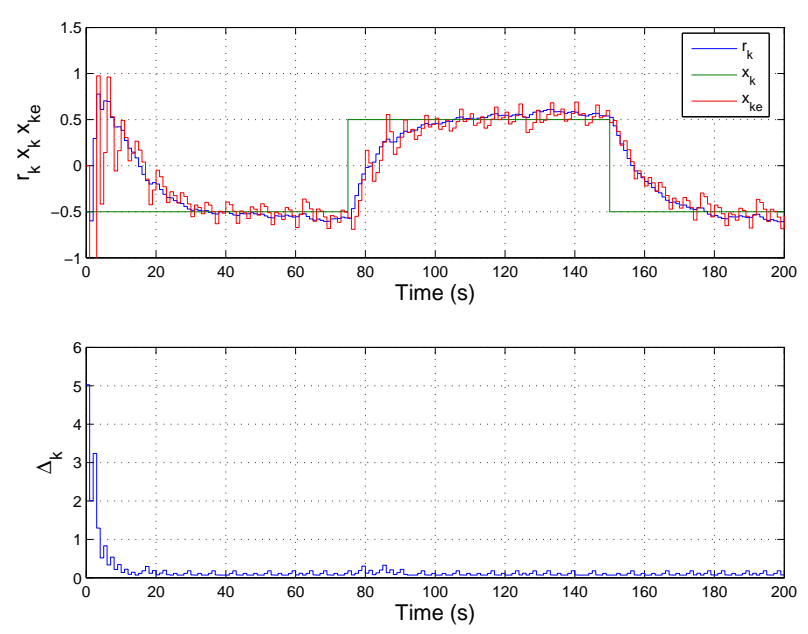

Fig. 6. Simulation results for adaptive $\Delta-M$ scheme.

samples would be required for (8) to make $\Delta_{k}$ large enough to capture again such state (i.e. the transient time would undesirably depend on the time the system spends on the steady state).

\section{CONCLUSIONS}

In this paper we have investigated the stability properties of the Delta-modulation coding rule, when used as a transmission mean in networked controlled linear systems. It was first shown that the standard form of the $\Delta-M$ algorithm can be modified, including information about the system and the controller. These results were extended to the case of adaptive $\Delta_{k}$. An explicit adaptation rule was proposed and range of parameters were derived to ensure asymptotic stability. These results displayed a limit on the maximum unstable eigenvalues of the system that are compatible with the ones given in [11].

\section{ACKNOWLEDGEMENTS.}

Special thanks are due to Prof. Chaouki Abdallah and Ivan Lopez for indicating the additional condition on parameter $a$ in Theorem 1. Thanks are also due to Prof. Sanjoy Mitter for his comments and discussion on the issue of this paper. The authors would like to acknowledge MCYT-FEDER for funding this work under grants DPI2004-06419, HF20030237 and SAB2003-0085. Thanks for funding are also due to the programm PICASSO No. 07261YJ(EGIDE), of the French Minister of foreign affaires.

\section{REFERENCES}

[1] Brockett R.-W. and Liberzon D. Quantized feedback stabilization of linear systems. IEEE Transactions on Automatic Control, 45(7):12791289 , July 2000.

[2] C. Canudas-de-Wit, F. R. Rubio, J. Fornes, and F. Gomez-Estern. Differential coding in networked controlled linear systems. To appear in the American Control Conference. Silver Anniversary ACC. Minneapolis, Minnesota USA, 2006.

[3] Elia N. and S.-K. Mitter. Stabilization of linear systems with limited information. IEEE Transaction on Automatic Control, 46(9):13841400, September 2001.

[4] Hespanha J.-P., Ortega A., and Vasudevan L. Towards the control of linear systems with minimum bit-rate. In 15th Int. Symp. Mathematical Theory of Networks and Systems (MTNS), Notre Dame, IL, USA, 2002.

[5] Ishii H. and T. Başar. Remote control of lti systems over networks with state quatization. System and Control Letters, (54):15-31, 2005.

[6] Lemmon M. and Q. Ling. Control system performance under dynamic quatization: the scalar case. In 43rd IEEE Conference on Decision and Control, pages 1884-1888, Atlantis, Paradice Island, Bahamas, 2004.

[7] Li K. and J. Baillieul. Robust quatization for diginal finite communication bandwidth (dfcb) control. IEEE Transaction on Automatic Control, 49(9):1573-1584, September 2004.

[8] Liberzon D. On stabilization of linear systems with limited information. IEEE Transaction on Automatic Control, 48(2):304-307, February 2003.

[9] Proakis J.-G. Digital Communications. McGraw-Hill, Inc. Series in electrical and computer enginering.

[10] Tan S., Xi Wei, and J.-S. Baras. Numerical study of joint quatization and control under block-coding. In 43rd IEEE Conference on Decision and Control, pages 4515-4520, Atlantis, Paradice Island, Bahamas, 2004.

[11] S. Tatikonda and S.K. Mitter. Control under communication constraints. IEEE Transaction on Automatic Control, 49(7):1056-1068, July 2004. 